Karen Andersen - Laura Rodríguez - Cristóbal Balbontín: Las mujeres en la reconstrucción del espacio público post-catástrofe socio-natural en Dichato, Chile (2010-2013). Una aproximación hermenéutica desde el mito de Antígona, Izquierdas, 49, abril 2020:725-742

\title{
Las mujeres en la reconstrucción del espacio público post-catástrofe socio- natural en Dichato, Chile (2010-2013). Una aproximación hermenéutica desde el mito de Antígona.
}

Women in the reconstruction of the public space post-catastrophe socionatural in Dichato, Chile (2010-2013). An hermeneutic approach in the perspective of Antigona's myth.

\author{
Karen Andersen Cirera* - Laura Rodríguez Negrete** Cristóbal Balbontín Gallo***
}

Resumen: A pesar de los avances en equidad de género en Chile, aún existe una brecha a saldar en relación a la participación ciudadana. Se plantea una reflexión en torno a la participación de la mujer en el espacio público. A través de una investigación cualitativa en un campamento de emergencia post-catástrofe, vemos como las mujeres abren nuevos y alternativos espacios públicos capaces de acoger demandas específicas. Se plantea que la participación ciudadana postdesastre no está considerando aspectos de género, aun cuando son las mujeres las más activas en contextos de precariedad y vulnerabilidad como es el caso de la emergencia postdesastre del terremoto y tsunami ocurrido en Chile en 2010.

Palabras claves: espacio público; participación ciudadana; enfoque de género; resiliencia postdesastre: hermenéutica.

\begin{abstract}
Despite advances in gender equity in Chile, there is still a gap to be settled in relation to citizen participation. A reflection is proposed regarding the participation of women in public space. Through qualitative research in a post-catastrophe emergency camp, we see how women open new and alternative public spaces capable of accommodating specific demands. It is argued that post-disaster citizen participation is not considering gender aspects, even though women are the most active in contexts of precariousness and vulnerability, as in the case of the post-disaster emergency of the earthquake and tsunami that occurred in Chile in 2010.
\end{abstract}

Keywords: public space; citizen participation; Gender approach; post-disaster resilience; hermeneutic.

Recibido: 11 diciembre 2018 Aceptado: 21 febrero 2019

\footnotetext{
* Chilena. Coautora. Doctora y Master en Urbanismo, Université Paris-Est, Francia. Académica Instituto de Arquitectura y Urbanismo, Universidad Austral de Chile. kandersenc@gmail.com

** Chilena. Coautora. Doctora en Ciencias Humanas, Master en Geografía, Master en Planificación Ambiental. Académica Instituto de Arquitectura y Urbanismo, Universidad Austral de Chile. Irodrigeocultural@gmail.com

*** Chileno. Coautor. Profesor Auxiliar Universidad Austral de Chile. Chercheur associé IREPH. Université Paris-Nanterre. cbalbonting@gmail.com

NOTA: Esta investigación fue financiada por Programa de Formación de Capital Avanzado y su Beca Master 2 Investigación y Doctorado Embajada de Francia en Chile - CONICYT otorgada a Karen Andersen Cirera.
} 
Karen Andersen - Laura Rodríguez - Cristóbal Balbontín: Las mujeres en la reconstrucción del espacio público post-catástrofe socio-natural en Dichato, Chile (2010-2013). Una aproximación hermenéutica desde el mito de Antígona, Izquierdas, 49, abril 2020:725-742

\section{Introducción}

Hace algo más de un año atrás, después de vivir el dantesco infierno de los incendios forestales en todo el centro-sur del país, el diario digital BBC se preguntaba ¿Qué hace que los chilenos sean tan resistentes a los terremotos, incendios y otras tragedias? ${ }^{2}$ Las respuestas no dejaron de llegar, desde las distintas ciencias sociales intentaron dar con la clave resolutoria del dilema planteado por el informativo. No obstante, en cada una de ellas, había un sujeto ausente; invisibilizado en el relato histórico, las mujeres han sido protagonistas activas en los procesos de reconstrucción frente a los desastres naturales, siendo sistemáticamente obliteradas del protagonismo que -en la práctica- asumen. Esta investigación, aborda la contribución de las mujeres en los procesos de reconstrucción postcatástrofe, discutiendo el papel que ellas asumen, incluso en terrenos donde han sido históricamente marginadas, tales como, la esfera pública. La exclusión de las mujeres, de la esfera pública, encuentra un asiento en los estratos más profundos de la civilización occidental, construido desde la época clásica de la cultura griega, donde el mito de Antígona, nos permite a partir de la interpretación de este mito, una explicación al fenómeno abordado en esta investigación.

Luego del terremoto y tsunami, ocurrido en Chile el 27 de febrero de 2010, el cual afectó aproximadamente a 2,5 millones de personas, con 8,8 grados en la escala Richter, en palabras de González "lo convierte en el sexto terremoto más grande del cual se tenga registro. Más de novecientas ciudades y localidades menores fueron afectadas"2. Siendo la Región del Biobío, una de las más afectadas por el terremoto y el tsunami, hace que siga teniendo una especial relevancia en los estudios relativos a los desastres socionaturales.

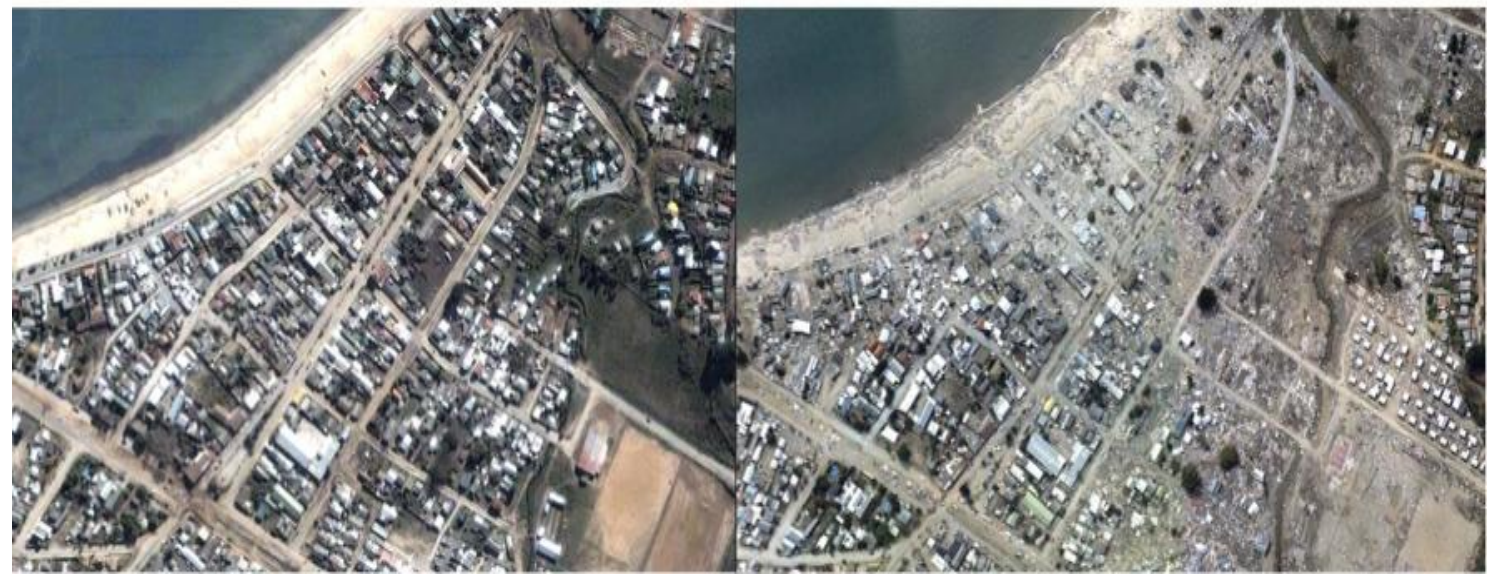

Imagen 1. Dichato antes y después del terremoto y tsunami 2010. Fuente: Google Earth.

Rápidamente se levantaron diversos campamentos para responder a la emergencia producida. Uno de los muchos asentamientos construidos fue "El Molino", en el pueblo de Dichato, Región del Biobío, donde se pudo observar la utilización de diversos mecanismos por los cuales la

\footnotetext{
1 Diario BBC Mundo, 2 febrero 2017

2 Claudia González-Muzzio, "El rol del lugar y el capital social en la resiliencia comunitaria posdesastre. Aproximaciones mediante un estudio de caso después del terremoto del 27/F”, Revista EURE. Vol 39, n 117, 2013:16
} 
Karen Andersen - Laura Rodríguez - Cristóbal Balbontín: Las mujeres en la reconstrucción del espacio público post-catástrofe socio-natural en Dichato, Chile (2010-2013). Una aproximación hermenéutica desde el mito de Antígona, Izquierdas, 49, abril 2020:725-742

comunidad fue tratando de recuperarse del desastre sucedido; uno de éstos fue la co-construcción de diversos tipos de espacios públicos por los habitantes, estos permitieron algo necesario y urgente para la sucesiva recuperación. El encuentro en la incipiente esfera pública del campamento fue permitiendo una embrionaria organización comunitaria, la cual podía en el mejor de los términos poner en movimiento la reconstrucción física, social y psicológica de la comunidad, que se dio en paralelo a la reconstrucción planificada desde el Estado y las instituciones privadas que comenzaron a sumarse al proceso. La investigación se llevó a cabo, entre los años 2010 y 2013, en este lugar.

La relevancia de evidenciar estos resultados surge de la necesidad de distinguir ciertos sujetos y espacios sociales, invisibilizados por las investigaciones post catástrofes, y que en nuestra opinión cumplen un rol significativo en la recuperación de los territorios, especialmente en el caso de Chile, donde esta investigación está situada.

Luego de un desastre socio natural, entendido como el fenómeno que se produce en la interrelación entre "lo natural" y "lo social"3; la reconstrucción de un pueblo y su territorio, apunta hacia la correcta articulación de sucesivas fases, siendo la primera, hacer frente a un estado de emergencia. Esto implica, restablecer la seguridad de los asentamientos, lo cual conlleva dar refugio y abastecimiento a las víctimas, especialmente las que han perdido a sus seres queridos y sus viviendas. Si bien la reconstrucción de los espacios públicos, dentro de la agenda prioritaria del Estado, queda ubicada en una posición subordinada a las anteriores, estos espacios son claves para la resiliencia comunitaria. ${ }^{4}$

Por otra parte, el desastre es vivido de manera diferente en función del género, la edad y de la situación económica y social de las víctimas. El reconocimiento de una identidad de género -que ha sido construida socialmente- implica en nuestra sociedad ciertos roles sociales específicos que son reclamados, impulsados, desde las diversas instituciones que operan en la situación de pos catástrofe.

El Gobierno de Chile organizó la reconstrucción a partir de un Plan de Reconstrucción Nacional, el cual a través de un Comité de Reconstrucción ponía en dialogo a diversas instituciones públicas5. Por otra parte, el Servicio Nacional de la Mujer, SERNAM, pone en acción el plan "Mujer, Levantemos Chile", que consistió en una serie de acciones que se implementaron en las regiones afectadas $^{6}$. Si bien estas acciones fueron importantes para apoyar a las mujeres a hacer frente a nuevos problemas, estas acciones fueron en gran parte ayudas económicas, traducidas en becas o bonos, no considerando una propuesta entorno a problemáticas de género como la violencia o de la salud reproductiva. Se destaca en este sentido la atención psicológica que se dio en algunos centros del SERNAM.

La contraposición entre la vulnerabilidad y la resiliencia femenina, observada en la investigación es dependiente de factores culturales, históricos, como también de orden económico, por lo que el análisis no está sujeto a reglas universalizantes y se restringe a ciertos atributos propios de sociedades, en este caso, en camino del desarrollo. ${ }^{7}$

\footnotetext{
3 Romero Toledo, Hugo, Romero Aravena, Hugo. "Ecología política de los desastres: vulnerabilidad, exclusión socio-territorial y erupciones volcánicas en la patagonia chilena”. Magallania (Punta Arenas), 43(3), 2015:10

${ }_{4}$ Allan, Penny y Bryant, Martin, "The Critical Role of Open Space in Earthquake Recovery : A Case Study", Paper n 34 NZSEE Conference (New Zealand Society for Earthquake Engineering Inc), Victoria University of Wellington, Wellington, New Zealand. URL: http://www.nzsee.org.nz/db/2010/,2010.

5 Ministerio de Obras Públicas, Ministerio de Vivienda y Urbanismo, Ministerio de Transportes y telecomunicaciones, Ministerio de Bienes Nacionales, Ministerio de Planificación, Secretaría General de la Presidencia, Ministerio de Salud y Ministerio de Educación.

${ }^{6}$ La aplicación de un programa de empleos, medidas de apoyo al emprendimiento a través de créditos blandos, tramitación de subsidios de vivienda para mujeres, un bono de emergencia agrícola, se instalaron salas con conectividad digital, se dieron becas de estudios y se financió la educación pre-escolar, se instaló una clínica móvil y vacunación gratuita, se realizó contención emocional en línea, a través de el programa "Salud Responde" y se dio atención psicológica especializada en algunos centros de la Mujer de SERNAM, además se realizaron charlas motivacionales. (SERNAM, "Balance Gestion Integral 2010", 2010).

7 ONU, Bureau pour la Prévention des Crises et le Redressement, Programme des Nations Unies pour le Développement (PNUD), Genre et Catastrophe. URL: www.undp.org, 2010.
} 
Karen Andersen - Laura Rodríguez - Cristóbal Balbontín: Las mujeres en la reconstrucción del espacio público post-catástrofe socio-natural en Dichato, Chile (2010-2013). Una aproximación hermenéutica desde el mito de Antígona, Izquierdas, 49, abril 2020:725-742

En un contexto de emergencia post-catástrofe, estudios realizados por el Programa de las Naciones Unidas para el Desarrollo (PNUD) en diversos países afectados por una catástrofe, nos confirma que las mujeres y las niñas son las más expuestas en una catástrofe natural. "Según los datos de 141 países afectados por desastres entre 1981 y 2002, los desastres tienen un impacto negativo mayor sobre la esperanza de vida de las mujeres que sobre la de los hombres. Las mujeres, los niños y las niñas son 14 veces más propensos que los hombres a morir durante un desastre. La mayoría de las víctimas atrapadas en Nueva Orleans después del huracán Katrina fueron mujeres afroamericanas y sus hijos, el grupo demográfico más pobre de la zona. Las probabilidades de que una mujer sea víctima de la violencia doméstica o sexual aumentan después de un desastre. Muchas mujeres evitan albergarse en refugios por miedo a ser violadas"8 En resumen, en la situación de crisis son más propensas a sufrir violencias al interior de los hogares. ${ }^{9}$

Por otro lado, según un estudio del Observatorio de Desarrollo Social del Gobierno de Chile, en Chile las mujeres jefas de familia han aumentado de un 20\% a un 39,5\% desde el año 1990 hasta el 2015 y dentro de los hogares liderados por una mujer, al año 2015 el 77,4\% son monoparentales. ${ }^{10} \mathrm{La}$ dificultad de acceso a un empleo que les permita conciliar sus responsabilidades domésticas y familiares con una actividad remunerada, lleva a que una gran parte de estas mujeres ejercen actividades informales y precarias, lo que las hace todavía más vulnerables. ${ }^{11}$

Según la ONEMI (Oficina Nacional de Emergencia de Ministerio del Interior del Gobierno de Chile) las mujeres enfrentan otras experiencias en el proceso de la emergencia, distintas a las que experimentan los hombres. Primero, en lo que se refiere a las mujeres dependientes, estas tienen menos recursos dentro del hogar, por lo que tienen menos poder de negociación sobre las decisiones de la familia. La catástrofe trae aumento en el desempleo, por lo que, en el caso de las mujeres jefas de hogar, muchas de ellas se ven sin fuente de ingresos. No obstante, las mujeres son las encargadas del aseo y la cocina en las viviendas de emergencia, tal como deben supervisar la organización de las ayudas. Adicionalmente, las mujeres se ocupan del cuidado de los niños, de los adultos mayores y de las personas enfermas o con discapacidad. Junto con lo anterior, ellas colaboran además con la red familiar y comunitaria, a la resolución de los problemas prácticos y afectivos.

Indudablemente las necesidades de las mujeres en la emergencia difieren de las de los hombres. Los tipos de ayudas y servicios requeridos por estas son muy específicos. Las restricciones de acceso a artículos de higiene femenina, la asistencia médica durante el embarazo o la anticoncepción, ejercen una presión a la condición biológica, situándola física y psicológicamente dentro de una mayor vulnerabilidad. Por último, la necesidad de generar un ingreso las lleva a desempeñar labores, que muchas veces se traduce en una sobrecarga de responsabilidades en una situación posterior al desastre. ${ }^{12}$

Paralelamente, la acción pública no considera esta diferencia al proponer, como respuestas a la emergencia, soluciones estándar, sin distinción de género. La planificación de la emergencia y de la reconstrucción en Chile no considera una perspectiva de género en sus intervenciones. Tampoco existe un adecuado diagnóstico sobre la construcción colectiva de la esfera pública, necesaria y urgente de ser incorporada dentro del proceso oficial de participación ciudadana.

\footnotetext{
8 Oficina de Prevención de Crisis y Recuperación, Programa de las Naciones Unidas para el Desarrollo, Genero y Desastres., www.undp.org. 2010.

${ }^{9}$ Consuelo Cornejo, Oficina Nacional de Emergencia Ministerio del Interior y Seguridad Pública División de Protección Civil, Reducción del Riesgo de Desastre con Enfoque de Género. Género, Desastres y Gestión. Programa de Mejoramiento de la Gestión Sistema de Equidad de Género. URL: www.onemi.cl, 2012.

10 Según los datos de la encuesta CASEN de los años 1990 y 2015.

11 Según el Documento "Enfoque estadístico de octubre 2018 sobre Género e informalidad Laboral" realizado por el Instituto Nacional de Estadísticas. Chile. Consultado en http://www.ine.cl/docs/default-source/laborales/informalidadlaboral/publicaciones/2018/enfoque-de-genero-e-informalidad-laboral-2018.pdf?sfvrsn=5

12 WHO, Department of Gender, Women and Health, Gender and Health in Natural Disaster. URL : http://www.who.int/genderequity-rights/en/, 2005; ONU, 2010; Cornejo, 2012.
} 
Karen Andersen - Laura Rodríguez - Cristóbal Balbontín: Las mujeres en la reconstrucción del espacio público post-catástrofe socio-natural en Dichato, Chile (2010-2013). Una aproximación hermenéutica desde el mito de Antígona, Izquierdas, 49, abril 2020:725-742

El presente artículo presenta primero una discusión teórica sobre la participación de la mujer en el espacio público, incorporando una perspectiva hermenéutica, donde se resalta, desde la tradición griega el rol de la mujer en la disputa del espacio. Posteriormente, ahonda en los resultados de una investigación cualitativa sobre la participación de la mujer en los espacios públicos del campamento de emergencia de Dichato. Finalmente, el artículo busca evaluar la brecha relacionada con las políticas de emergencia, y la inclusión de las potencialidades y necesidades de los espacios públicos femeninos.

\section{Metodología}

Esta investigación centra su enfoque en una metodología cualitativa, incorporando inicialmente el análisis hermenéutico -donde a partir del mito de Antígona- se analiza la obra griega escrita por Sófocles, donde ya podemos advertir el rol que le cabe a la mujer en la esfera pública. Por hermenéutica podemos entender el venir interpretado del mundo en el que cotidianamente nos movemos. ${ }^{13}$ Allí lo que pasa inadvertido para la mirada ingenua, y en consecuencia permanece inaccesible (bermeneia) ${ }^{14}$, el fenomenólogo lo logra advertir como narrativa ${ }^{15}$ que cruza como un tejido las relaciones entre las cosas, configurando un orden de sentido al interior del cual también se ven envueltos los seres humanos. Por su parte, el enfoque etnográfico permite comprender el espacio público cotidiano de un campamento de emergencia, cobrando especial relevancia la profundidad y envergadura de los relatos, facilitando con esto una comprensión más amplia del fenómeno. La metodología incorpora además el Estudio de Caso, el cual se basa en la observación de campo y entrevistas en el campamento de emergencia "El Molino" de Dichato durante los años 2011 y 2013.

Nuestro Caso de Estudio corresponde al campamento de emergencia más grande y poblado del país luego del terremoto y tsunami de Chile el año 2010, y se encuentra ubicado en la localidad de Dichato, en la comuna de Tomé, Región del Biobío, Chile. El enfoque etnográfico, en el campamento "El Molino", busca describir la realidad vivida y percibida por las mujeres habitantes de un campamento de emergencia. A causa de la difícil situación experimentada por los habitantes de este campamento, se consideró necesario avanzar en el trabajo de campo en dos etapas; Una primera etapa de impregnación ${ }^{16}$, donde durante el transcurso del mes de abril del año 2011, se realizaron visitas diarias de observación y de conversaciones informales y sin grabadora, con el doble propósito de tener un primer acercamiento a los problemas del campamento y familiarizar a los habitantes con la presencia del investigador. Durante este tiempo, se advirtió la necesidad de las mujeres de ser escuchadas, por lo que fue de suma importancia trabajar el proceso de generar confianzas con las potenciales entrevistadas. En una segunda etapa, se continuó con un periodo de observación, pero además se fueron incluyendo la realización de entrevistas semiestructuradas en profundidad, lo cual se realizó durante dos semanas, en el mes de abril y dos semanas en el mes de mayo del año 2011. ${ }^{17}$ La observación nos informó de elementos concretos de la configuración y organización espacial, así como las formas y ritmos de frecuentación y usos de los espacios comunes en el cotidiano. Las entrevistas realizadas correspondieron a entrevistas en profundidad semiestructuradas. Se realizaron un total de 11 entrevistas a mujeres residentes en el campamento "El Molino" de Dichato, las cuales nos permitieron entender aspectos más sensibles de la relación entre el

\footnotetext{
13 Martin Heidegger, Sein und Zeit, Tubingen: Niemeyer, 1926.

14 Recordemos que Hermes era el Dios del secreto.

15 Paul Ricoeur, De l'interpretation, Paris, Editions Seuil, 1965.

16 Michèle Grosjean y Jean-Paul Thibaud, (ed), L'espace public en méthodes, Editions Parenthèses, 2008.

${ }^{17}$ Daniel Cefai, L'Enquête de terrain, Paris, Editions La Decouverte. 2003.
} 
Karen Andersen - Laura Rodríguez - Cristóbal Balbontín: Las mujeres en la reconstrucción del espacio público post-catástrofe socio-natural en Dichato, Chile (2010-2013). Una aproximación hermenéutica desde el mito de Antígona, Izquierdas, 49, abril 2020:725-742

espacio común y el espacio privado. Las entrevistas fueron realizadas a mujeres de edades y contextos familiares diferentes ${ }^{18}$.

\section{Hermenéutica de la participación de las mujeres en el espacio público}

En la historia de las sociedades y de los Estados, la esfera pública es definida por los espacios que dan visibilidad, debate y solución a los temas de interés común a la sociedad. No obstante, desde los griegos, la esfera pública se ha construido sobre principios masculinos. Las mujeres, junto a los extranjeros y los pobres, han sido marginados durante siglos, alejados de los espacios de participación e importantemente de los espacios de decisión. Tal es el caso, por ejemplo -siguiendo a Simone de Beauvoir ${ }^{19}$ y Honig ${ }^{20}$ - de la exegesis feminista de uno de los mitos fundacionales en la Grecia antigua: Antígona de Sófocles, tal como lo presenta Hegel en su Fenomenología del espiritu²1.

En efecto, Antígona representa la anarquía y la resistencia, cuando el amor sagrado de la familia, del ámbito de la naturaleza y de lo privado se ve amenazado por la ley positiva de la polis, a causa de la expresión de lo privado en el espacio público. Después del exilio del rey Edipo, sus hijos, Eteocles y Polinices, se enfrentan por el trono de la ciudad de Tebas. Los hermanos mueren producto de esta batalla. Creonte, hermano de Edipo, después de la muerte de los hermanos, asume el trono de Tebas. Creón da a Eteocles los honores funerarios, negándole los mismos a Polinices. Antígona, hija de Edipo se revela contra esta decisión apelando a la justicia divina, a la piedad y al respeto por la memoria de sus hermanos y de su familia, y decide rendirle honor a su hermano Polinices con los homenajes funerarios. Antígona, desobedeciendo la ley del rey Creonte, y revelándose contra su poder despótico, va a recibir un terrible castigo. Esta acción moral de deferencia hacia los muertos y los dioses va a implicar su sacrificio. Creonte condena a Antígona al encierro eterno. Antígona por su parte escogerá la muerte sobre la obediencia.

El mito de la Antígona, de Sófocles, presentaría, de esta forma, la dialéctica de la ley humana y la ley divina a través de la oposición entre Creonte y Antígona. La tensión y la oposición nacidas de la decisión de Antígona contra la ley de los hombres, con el fin de darle sepultura a su hermano Polinices, corresponde a la tensión y a la oposición entre la ética que impone la familia contra la ética de las leyes escritas por los hombres. Antígona perturba el orden establecido de las leyes humanas a través de una acción impulsada por un deseo íntimo y de orden divino: la muerte. Para Creonte, los asuntos personales no pueden ser políticos. Solo el hombre accede a la universalidad, a la polis, él representa la cultura y funda las leyes. La mujer, es el objeto de su deber ético, ella se confía a la familia para desviar las ambiciones públicas, hacia fines privados. Cuando la mujer produce esta desviación, la ley la reprime, es por eso que Creonte va a matar a Antígona.22

Tal oposición de lo público/privado también está presente en autores como Rousseau, quien en su texto Emilio o De la educación, exalta los rasgos de autonomía y emancipación de la educación de Emilio en el rito de pasaje que marca la educación, para transitar desde la familia a la sociedad. Mientras que la educación de Sofía -quien ocupa una posición marginal en su obra- es funcional y complementaria a la vida pública que llevará Emilio23.

\footnotetext{
18 Las entrevistas fueron realizadas a las siguientes residentes del campamento : Macarena, 40 años, dirigente y madre ; Eliana, 60 años, abuela y emprendedora ; María, 50 años, madre y emprendedora, Carolina, 45 años, madre, trabajaba en la sede comunitaria; Carmen, 60 años, trabajaba con adultos mayores del campamento ; Marisa, 50 años, abuela y ex-dirigente ; Tatiana, 35 años, dirigente, emprendedora y madre ; Pilar, 25 años, artesana, vive con su abuela ; Eduvina, 80 años, abuela con discapacidad visual ; Margarita, 85 años, vivía sola, Claudia, 40 años, vivía sola.

${ }_{19}$ De Beauvoir, Simone, The Second Sex. London: Jonathan Cape, 1956, p.581

${ }^{20}$ Honig, Bonnie. Antigone Interrupted. New York: Cambridge University Press, 2013, p.10

${ }^{21}$ Hegel, G.W.F. Werke 3: Phänomenologie des Geistes. Frankfurt, Suhrkamp.1989.

${ }^{22}$ Celia Amoros, Feminismo, igualdad y diferencia, México, UNAM, PUEG, 1994.

${ }^{23}$ Rousseau, Jean-Jaques, Émile ou De l'éducation, Paris,Garnier, 1961.
} 
Karen Andersen - Laura Rodríguez - Cristóbal Balbontín: Las mujeres en la reconstrucción del espacio público post-catástrofe socio-natural en Dichato, Chile (2010-2013). Una aproximación hermenéutica desde el mito de Antígona, Izquierdas, 49, abril 2020:725-742

No obstante, interpretaciones feministas posteriores de la presentación hegeliana del mito de Antígona han variado de dirección, desde el deseo de inclusión en lo público y la inclusión social a la radicalización de las diferencias ${ }^{24}$. En lo que importa, Butler dirá al respecto del mito de Antígona:

Las ironías son, sin duda alguna, mucho más profundas de lo que Hegel las entendió: después de todo, ella habla y lo hace en público, precisamente cuando hubiera debido estar recluida en el ámbito privado. ¿qué clase de discurso político es éste que transgrede las mismas fronteras de lo político, que pone escandalosamente en cuestión los límites que debieran determinar su discurso?25

La idea de un espacio público que acepta la expresión de la diversidad de opiniones, se basa en la teoría de la filosofía política desarrollada en los años 1960 por Habermas y Arendt. Sin embargo, al igual que en la polis, este espacio público liberal excluye a ciertos grupos de la sociedad, entre ellos, las mujeres. Incluso si Habermas reconoce espacios públicos alternativos al de su estudio, como la esfera pública plebeya, Nancy Fraser critica la idealización de esta esfera pública liberal: "En la medida que la puesta entre paréntesis de las desigualdades sociales en la deliberación significa proceder como si no existieran- cuando si existen - esto no ayuda, no estimula una paridad participativa". ${ }^{26}$ Fraser deplora el hecho que Habermas glorifique la esfera pública ignorando las otras esferas con las cuales esta concurre, limitándose al análisis de un solo espacio público. ${ }^{27}$

El espacio público es el espacio de reconocimiento, de la competencia, contrariamente a las actividades del espacio privado que no son visibilizadas, ni valorizadas públicamente. En el espacio privado, no se expresa ningún principio de individuación, característica de los espacios públicos donde cada uno tiene su $u b i$ (donde). En la apropiación de los espacios por múltiples individualidades, el poder se distribuye. ${ }^{28}$ Así, el espacio privado adquiere el estatus de zona escondida, donde no existe ni reconocimiento ni individuación. Así, sin reconocimiento no hay existencia social.

No obstante, la mujer y su emocionalidad, frente a la experiencia de la injusticia irrumpen en el espacio político de los años 1960, tal como Antígona irrumpe en el mundo político griego, blandiendo una reivindicación privada. Con esta irrupción, Bondi remarca el desafío planteado por las geógrafas feministas respecto de las categorías binarias asociadas a la masculinidad y la racionalidad, y por ende la objetividad y la femeneidad asociada a la emocionalidad, el cuerpo y la subjetividad, desestabilizando esta dualidad. ${ }^{29}$

El espacio público, considerado hasta entonces como exclusivamente masculino, entra hoy en conflicto con el espacio femenino, que supone ser más familiar y emotivo, pero siempre público, por el hecho de ser asunto de toda una sociedad. Hasta la segunda mitad del siglo XX, el espacio público ciudadano, las instituciones y el poder se mantenían mudos frente a una sociedad en la cual la mujer se hacía cada vez más activa y se beneficiaba progresivamente de derechos. Las feministas americanas de los años 1960 y 1970, a partir de su eslogan "lo personal es político" o "lo privado es político" abren el debate público. La esfera pública acepta asuntos del orden de lo íntimo y de lo doméstico que hasta entonces eran invisibles, desplazados del debate. Primero, el movimiento feminista logra introducir la resolución de algunos asuntos privados en la agenda de las decisiones públicas. Después, ellas logran politizar el movimiento y legislar sobre diversos temas hasta ahora "asuntos femeninos" como la maternidad, la concepción o la violencia doméstica.

\footnotetext{
${ }^{24}$ Este giro hermenéutico toma especialmente como hito la obra de: Irigaray, Luce. Speculum of the Other Woman. New York: Cornell University Press, 1987.

25 Butler, Judith. El Grito de Antígona. Barcelona; El Roure Editorial, 2001:19

${ }^{26}$ Nancy Fraser, "Rethinking the Public Sphere: A Contribution to the Critique of Actually Existing Democracy", Social Text, No. 25/26, pp. 56-80 Published by: Duke University Press Stable. URL: http://www.jstor.org/stable/466240. $1990: 64$.

27 Fraser, 1990.

28 Amoros, 1994.

${ }_{29}$ Bondi, Liz, "Making connections and thinking through emotions: between geography and psychotherapy". Trans Inst Br Geogr NS 30. 2005. Pp. 433-448
} 
Karen Andersen - Laura Rodríguez - Cristóbal Balbontín: Las mujeres en la reconstrucción del espacio público post-catástrofe socio-natural en Dichato, Chile (2010-2013). Una aproximación hermenéutica desde el mito de Antígona, Izquierdas, 49, abril 2020:725-742

Hanna Arendt y Celia Amorós constatan que la distinción existente en las representaciones entre lo público y lo privado impone a la mujer limitaciones en su participación política. ${ }^{30}$ Así, cuando esta distinción es perturbada, la mujer participa activamente del espacio público. Nosotros remarcamos a través de nuestra investigación de terreno, como en situaciones de trastorno de la vida privada, la mujer abre un espacio público alternativo, en ruptura con el diktat de un espacio público masculino.

\section{Etnografía de un campamento de emergencia. Historias femeninas de reconstrucción: El caso de estudio: Campamento "El Molino" de Dichato.}

Los campamentos de emergencia o "aldeas" fueron construidos por el Programa de Reconstrucción del Ministerio de Vivienda y Urbanismo del Gobierno de Chile para albergar a las víctimas, en todas las localidades afectadas por el terremoto y tsunami de febrero del 2010. Solamente en la región del Biobío se construyeron 76 aldeas, de las cuales 11 corresponden a la comuna de Tomé. El campamento "El Molino" es uno de 3 campamentos que se construyeron en la localidad de Dichato. Este campamento llegó a tener un total de 519 viviendas con 2.850 personas, siendo uno de los más densos del país (Universidad de Concepción, 2010). Este campamento se emplazó a un kilómetro del pueblo, en un sitio en altura con respecto al nivel del mar, aledaño a la ruta 0-014 que conecta Dichato con la localidad de Tomé, sobre terrenos forestales privados que fueron arrendados por el Gobierno de Chile. La distancia, pendiente y estado de la ruta entre el campamento y Dichato hacían difíciles los desplazamientos cotidianos.

La construcción del campamento "El Molino" y la instalación de las víctimas se hicieron en seis etapas, lo cual posteriormente se tradujo en sectores; cada uno con un espacio común abierto, una sede social, contenedores de baños comunes, lavaderos y bidones de agua. Afín de facilitar la organización, también permitió mantener un control de los habitantes. Si bien no había límites físicos entre un sector y otro, los habitantes del campamento podían reconocer cotidianamente, nombrando cada uno por el número correspondiente a su etapa. Del punto de vista de la estructura social, estos sectores sin embargo no coincidían con los barrios de origen de la población, y solo algunos pocos tuvieron la oportunidad de escoger sus vecinos.

Los espacios abiertos del campamento eran de libre acceso para todos, sin embargo, existía una diferenciación entre las calles y los espacios centrales de cada uno de los sectores. El reducido perfil de algunas calles, hizo que estas fueran apropiadas por extensiones de las viviendas o patios privados. No obstante, los espacios centrales, eran espacios de dimensiones generosas, pero sin ningún tipo de diseño urbano, vegetación o mobiliario. Producto del suelo de tierra, la falta de red de agua potable en las viviendas y la gran cantidad de animales portadores de enfermedades que circulan en los espacios comunes. La higiene y las condiciones sanitarias del lugar fueron siempre una amenaza para los habitantes.

\footnotetext{
30 Bethsabé Andía Pérez, "Lo Personal es Político : Una lectura de lo Público y lo Privado", Boletín Generando del Instituto Runa de Desarrollo y Estudios sobre Género, Año 1, nº10, Lima. 2007.
} 

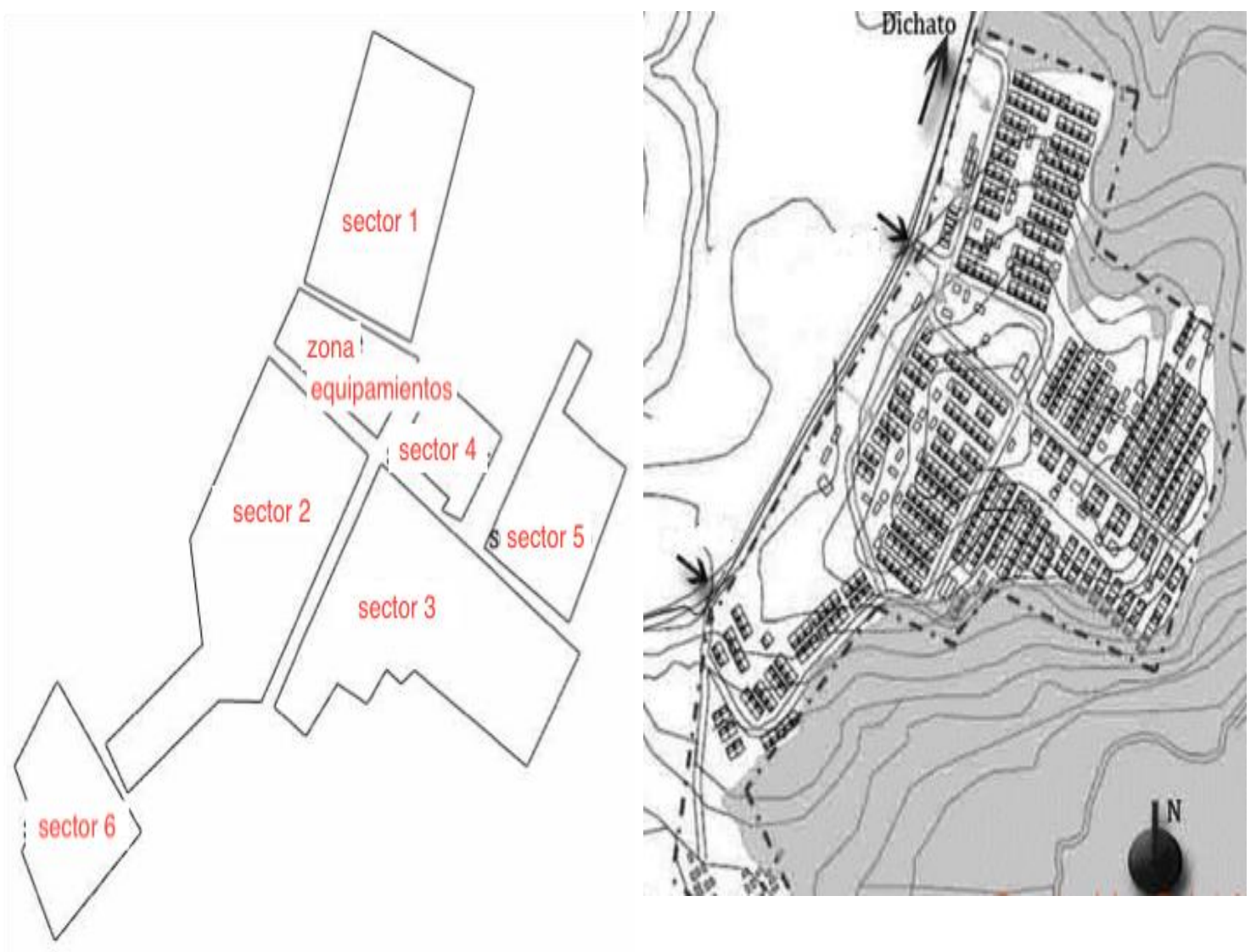

Imagen 2. Plano Campamento "El Molino”, etapas-sectores del campamento.

\section{El trabajo de las mujeres del campamento "El Molino".}

A partir de las entrevistas realizadas, se observa como antes del tsunami, las mujeres trabajaban principalmente en trabajos informales, dando alojamiento, trabajando en restaurantes, en la recolección de algas o en pequeños emprendimientos ocasionales. Las mujeres dichatinas, estaban acostumbradas al trabajo remunerado, especialmente durante la temporada de verano. Se desempeñaban en trabajos ligados a la agricultura, la cosecha de algas marinas y el comercio informal. Sin embargo, fueron justamente estos trabajos los más perturbados por el desastre natural, dejando a muchas mujeres desempleadas.

Todas las mujeres entrevistadas que trabajaban antes de la catástrofe, habían perdido sus trabajos, y en la situación de emergencia intentaban encontrar actividades remuneradas en medio de la escasez generalizada de ofertas de trabajo. Debido al declive del turismo en el balneario, producto del desastre natural, encontrar un trabajo en Dichato se hizo muy difícil. Además, al estar al cuidado de sus hijos y personas mayores, estas mujeres tenían un margen de movilidad mucho menor para encontrar una labor remunerada fuera del pueblo. En estas condiciones, la mujer trabajaba no solo para ella, sino especialmente para todos aquellos bajo su responsabilidad. En este contexto, sin duda tenía menos flexibilidad para aprovechar las posibles oportunidades de empleo. La cantidad de tiempo que se pasaba en los campamentos de emergencia empeoraba la vida cotidiana; de hecho, en la mayoría de los casos, 
Karen Andersen - Laura Rodríguez - Cristóbal Balbontín: Las mujeres en la reconstrucción del espacio público post-catástrofe socio-natural en Dichato, Chile (2010-2013). Una aproximación hermenéutica desde el mito de Antígona, Izquierdas, 49, abril 2020:725-742

las mujeres no tenían una actividad fuera del campamento. Tal como lo documentan, Detraz \& Peksen ${ }^{31}$ en un estudio internacional respecto de la pérdida de derechos humanos de las mujeres en contextos de desastres y entre ellos la vulnerabilidad económica en la cual se sitúan.

Por el contrario, los hombres caminaban todas las mañanas hacia la ruta, a la parada de autobús, para ir a las localidades cercanas o bien, se desplazaban a Dichato para ocupar su jornada en trabajos remunerados. Según la Organización Mundial de la Salud de las Naciones Unidas, el "éxodo de hombres" es común después de los desastres. ${ }^{32}$ El campamento en el día estaba deshabitado de hombres. En consecuencia, la mayor parte del día, los espacios del campamento eran solo cruzados por mujeres, por niños o adultos mayores. No se realizaba ningún trabajo dentro del campamento, el ambiente es residencial, a excepción de la instalación de algunos almacenes improvisados en algunas viviendas. Una mujer del campamento, cuyo esposo es albañil, nos dice:

Los hombres se van a las 6:30. A esa hora todos los hombres van a trabajar. A las 7 pm, vuelven. A partir de ahora, todos los lugares de actividades están muy lejos, si trabajan en el mar, deben bajar, si son trabajadores de la construcción, las construcciones los obligan a dejarlo todo. (Entrevista a Sra. Carolina, 45 años).

Las mujeres entrevistadas se encontraban intensamente involucradas en los asuntos públicos de la vida cotidiana de la comunidad y las víctimas del campamento "El Molino", donde pasaban la mayor parte de su tiempo. En la ausencia de servicios públicos después del desastre, las mujeres "reemplazaron" algunas de las instituciones de servicio. La mujer soportaba diariamente todas las limitaciones de la vida cotidiana del campamento: sacar el agua del exterior, lavar los platos y la ropa en los lavaderos comunes, lidiar con la tierra y la insalubridad de los perros vagos en los espacios comunes, limpiar los baños compartidos y aceptar las consecuencias de la proximidad con los vecinos.

Durante las entrevistas, las mujeres revelaron que habían pasado por estados depresivos después del desastre. Algunas mujeres todavía estaban siendo tratadas en el Hospital de Dichato por depresión. Durante los primeros meses, salieron muy poco de su hogar. Carmen, que trabajó todos los días desde agosto de 2010 como parte de un programa para personas mayores en el centro comunitario Hogar de Cristo, ubicado dentro del mismo campamento, nos da testimonio de la atmósfera de los primeros meses en "El Molino":

Cuando llegué, respiré el aire y supe... comencé a trabajar con una mujer española que se presentó como voluntaria aquí... cuando ingresamos al campamento, percibimos una depresión. Hubo tal silencio, los niños no salían a jugar, los adultos tampoco salían... nadie. (Entrevista a Sra. Carmen, 60 años)

Hemos podido constatar la fragilidad de los estados emocionales de las mujeres. Los silencios y lágrimas en las entrevistas, la tristeza en sus voces durante sus relatos, reflejan sus estados emocionales. Su sensación de soledad por la pérdida de redes y quiebre en las familias, así como el sentimiento de abandono por parte de las instituciones, se expresó fuertemente durante las entrevistas. Las mujeres nos relataron también los dramas y las tragedias de los otros vecinos. Tanto los espacios de copresencia, como los espacios de encuentro social son esenciales para escapar del aislamiento peculiar de este estado psicológico. Frente a esta vulnerabilidad Butler indica:

Así pensar el valor de la vulnerabilidad ha sido importante para la teoría feminista y la política, esto no significa que la vulnerabilidad sirva como una característica que define a las mujeres como

31 Detraz, Nicole, Peksen, Durson. "In the Aftermath of Earth, Wind, and Fire: Natural Disasters and Respect for Women's Rights”. Human Rights Review, 18(2), 2017. Pp.151-170. https://doi.org/10.1007/s12142-016-0440-4

32 WHO, 2005. 
Karen Andersen - Laura Rodríguez - Cristóbal Balbontín: Las mujeres en la reconstrucción del espacio público post-catástrofe socio-natural en Dichato, Chile (2010-2013). Una aproximación hermenéutica desde el mito de Antígona, Izquierdas, 49, abril 2020:725-742

grupo. Yo me opondría a este esfuerzo de manera de instalar una nueva norma para las categorías de las mujeres que descansa en una noción fundacional de vulnerabilidad. ${ }^{33}$

\section{Reconstrucción de la memoria del lugar.}

En la espera de que sus condiciones de vida volvieran a la normalidad, las mujeres intentaban en la medida de lo posible, reproducir los estilos de vida que tenían antes del desastre. La pérdida total de sus bienes, las llevaba a reproducir su habitar a través de intervenciones. Pudimos observar como la mediagua fue el soporte para algunas de estas intervenciones. Las mediaguas, que correspondieron a las casas de emergencia entregadas en los campamentos, son construcciones rectangulares de $18 \mathrm{~m} 2$ construidos con paneles de madera. Estas mediaguas se encontraban emplazadas una al lado de la otra sin espacios de antejardín. Los habitantes del campamento poco a poco empezaron a reconstruir sus formas de habitar, a través de incorporación de vegetación, huertas, bancas, mesas, lavaderos, construcción de accesos, cubiertas, terrazas, etc.

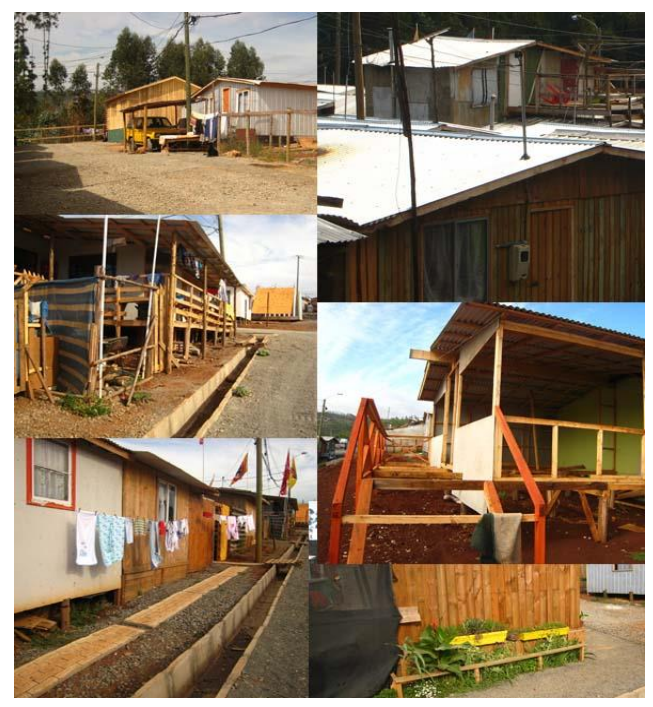

Imagen 3.- Construcciones y mejoramientos a la vivienda de emergencia realizados por los habitantes. Fuente: Karen Andersen, 2011.

En relación a la vida comunitaria, las mujeres del campamento de "El Molino" en Dichato jugaron un papel importante en la reactivación de la vida pública de las víctimas. Durante los primeros meses después de la destrucción del pueblo y las casas, la mala atmósfera moral, resultante de la tristeza y del miedo, había llevado a las víctimas a permanecer enclaustradas dentro de las casas, separándose del contacto con el exterior. La vida comunitaria que existía en Dichato se reactivó de otras formas al interior de los campamentos gracias a los espacios públicos creados por los habitantes, los cuales sirvieron como lugares de reunión de las víctimas para el intercambio y difusión de la información relevante de la emergencia y

reconstrucción.

\section{La gestión de lo doméstico en el espacio público}

Durante este período, las mujeres se dedicaron a varias tareas colectivas. Aseguraban las comidas comunes, la distribución de la ayuda y de los recursos externos entre la comunidad; la preocupación cotidiana de las personas mayores que vivían solas, la limpieza y la recolección de basura de los espacios comunes, el cuidado de los niños de madres que debían trabajar fuera del campamento, así como de velar por dar respuesta a toda necesidad o demanda que viniera de las víctimas. En la lectura de Antígona que realiza Caldarone ${ }^{34}$ dialoga perfectamente con esta idea de una subjetividad femenina muy lejos de la tradicional pasividad con que se las suele representar.

\footnotetext{
33 Butler, Judith, "Bodily Vulnerability, Coalitions, and Street Politics”. Critical Studies Vol. 37, 2014:110.

34 Caldarone, Rosaria. "Subjectivity and Femininity: Reading Antigone”. CR: The New Centennial Review, 17(2), 2017. Pp. 1-14. https://doi.org/10.14321/crnewcentrevi.17.2.0001
} 
Karen Andersen - Laura Rodríguez - Cristóbal Balbontín: Las mujeres en la reconstrucción del espacio público post-catástrofe socio-natural en Dichato, Chile (2010-2013). Una aproximación hermenéutica desde el mito de Antígona, Izquierdas, 49, abril 2020:725-742

Estos primeros espacios abiertos por grupos de mujeres a partir de dar respuesta a necesidades domésticas y cotidianas, abrieron espacios de participación alternativa de los habitantes al proceso de reconstrucción. Las víctimas demandaban apoyo para los problemas reales que enfrentan en la emergencia, así como de soluciones de reconstrucción atingentes a las realidades de las víctimas. De esta manera, las mujeres del campamento fueron conociendo las necesidades y sufrimientos que vivían los distintos grupos familiares, y comienzan a dialogar con las autoridades públicas. En estas circunstancias es donde el mito de Antígona cobra valor, donde el dolor emocional llevado en privado, hace gritar a Antígona, texto de Butler, en el cual encontramos que Antígona, no solo entierra a su hermano, en contra de lo decretado por Creonte, sino también, frente al requerimiento de este, ella responde "yo digo que lo hice y no lo niego". Las mujeres del campamento, expresan en estas instancias que van creando como públicas ese dolor íntimo de la esfera privada.

Cabe señalar que, si bien nos centramos en el trabajo de los grupos de mujeres, muchos hombres se convirtieron también en líderes de estos grupos constituidos principalmente por mujeres. Un ejemplo de esta participación ciudadana, se gestó por la denuncia de falta de claridad con respecto a las soluciones de reconstrucción, así como por la vulnerabilidad resentida en el campamento "El Molino", lo que llevó en un principio a una serie de manifestaciones en la ruta de acceso al pueblo, y luego a la creación del Movimiento ciudadano Asamblea de Dichato, movimiento que luego se sumó a el "Movimiento Nacional por una Reconstrucción Justa", movimiento ciudadano mayor conformado por víctimas de la catástrofe y por organizaciones sociales.

\section{Emprendimientos y gestión colectiva}

La solidaridad, el bienestar de los niños y los enfermos, la creación de espacios de trabajo, son algunas de las habilidades desarrolladas por las mujeres chilenas para hacerse cargo de la gestión de crisis. Esta experiencia es similar en muchas localidades afectadas por crisis y desastres. En este contexto, las mujeres han demostrado ser actores clave en la renovación de la vida pública, esta noción de vida pública no solo se entiende como el encuentro político, sino que también designa el placer de la reunión. Debido a la falta de espacios públicos de calidad en el campamento, los centros comunitarios se convirtieron en refugios seguros donde las víctimas se reunían. También a través de la organización de los vecinos, lograron ofrecerles a los niños un lugar para hacer sus tareas, con acceso a una computadora. Con una subvención de UNICEF, las sedes también ofrecieron una biblioteca con préstamo no solo de libros sino también de juegos y juguetes. Estas ludotecas, así como la supervisión de los niños al interior de las sedes, fueron proporcionadas por mujeres voluntarias, también víctimas. Siendo este aspecto de vital importancia de acuerdo a Berroeta, Ramoneda y Opazo ${ }^{35}$ para los procesos de reconstrucción.

Las mujeres del campamento gestionaron en el espacio de las sedes, una oferta de actividades y talleres para los habitantes del campamento. Estos talleres eran espacios de capacitación, que tenían como objetivo de transmitir conocimientos y habilidades a la población para encontrar empleos. Sin embargo, estos fueron también oportunidades para compartir y sobrellevar los sentimientos de soledad y los estados depresivos. Así, estos talleres se volvieron esenciales para que una población se encontrara con otros, ya que los espacios de recreación que frecuentaba antes de la catástrofe ya no existían, o bien, la tragedia había cambiado la relación emocional con ellos. Algunas personas manifestaban que no tenían deseos de ir al pueblo o a la playa, ya que estas visitas les generaban demasiada tristeza por los recuerdos que les suscitaban.

35 Berroeta, Hector, Ramoneda, Álvaro, Opazo, Luis, "Sentido de comunidad, participación y apego de lugar en comunidades desplazadas y no desplazadas post desastres: Chaitén y Constitución”, Universitas Psychologica, 14(4), 2015. Pp.1221-1233. https://doi.org/10.11144/Javeriana.up14-4.scpa . 
Karen Andersen - Laura Rodríguez - Cristóbal Balbontín: Las mujeres en la reconstrucción del espacio público post-catástrofe socio-natural en Dichato, Chile (2010-2013). Una aproximación hermenéutica desde el mito de Antígona, Izquierdas, 49, abril 2020:725-742

También notamos la existencia de iniciativas de emprendimiento de grupos particulares. Es el caso de un grupo de mujeres que construyó un invernadero en una de las áreas del campamento "El Molino" para cultivar y vender vegetales frescos. Este espacio se convirtió rápidamente en un lugar en sí mismo, cargado con una identidad particular y simbolismo para los habitantes del campamento. Aunque el objetivo del invernadero era convertirse en una fuente de trabajo lucrativa, el objetivo fue sobrepasado, ya que el trabajo y tiempo invertido en su construcción y mantención fueron además oportunidades para compartir experiencias y crear lazos humanos. El invernadero resultó un espacio abierto a todos, pero una regla tácita reserva el usufructo de los productos del invernadero a las mujeres que lo explotan.

\begin{abstract}
Mostramos con esto (el invernadero) que éramos capaces y también que podíamos salir de nuestras depresiones. Porque todas estábamos deprimidas, incluso cuando estábamos construyendo el invernadero y cultivando la tierra. Entonces el trabajo nos dio la oportunidad de conocernos, de reír... compartimos las tareas... una mujer estaba trabajando en el compost, otra estaba cavando la tierra, otra estaba haciendo otra cosa... en los descansos nos fumábamos un cigarrillo, nos reíamos y hablamos de todo, de cómo habían huido del tsunami, de todo lo que vivimos. (Entrevista a Sra. Eliana, 60 años).
\end{abstract}

Imagen 4. Invernadero colectivo- Fuente: Karen Andersen, 2011.

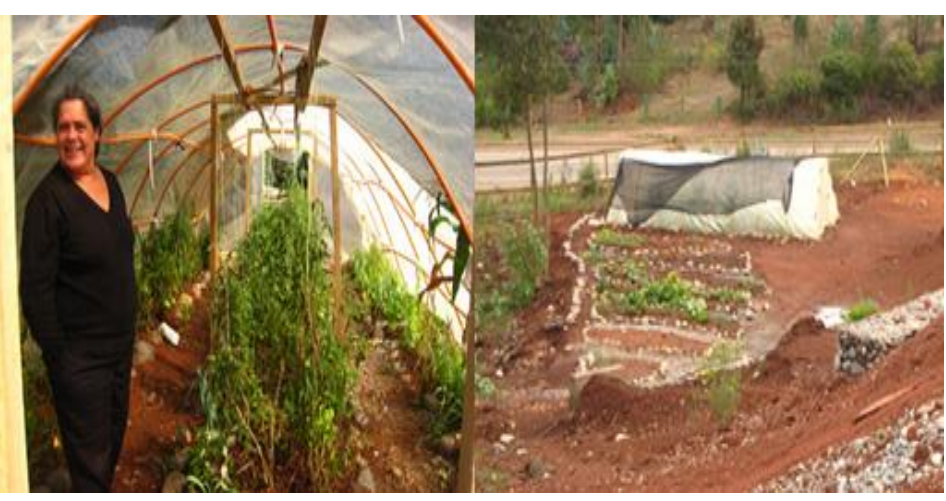

El trabajo colectivo, iniciado en los talleres de las sedes y en el marco de iniciativas aisladas emprendidas por grupos de habitantes, creó espacios de actividades colectivas, de intercambio, y dónde la información circulaba. Socializar sus preocupaciones y demandas a través de espacios de intercambio, en el contexto de actividades colectivas, ayudó a las víctimas a superar sus depresiones. Una de las motivaciones esenciales presentes en los discursos de las mujeres entrevistadas, que se movilizaron para recrear espacios colectivos era la restauración del bienestar de los niños, ya que los padres percibían la vida en el campamento como una amenaza para la continuidad de la educación de sus hijos. Antígona nos enseña que cuando el ámbito de lo privado se ve amenazado o negado por la ley, las mujeres son capaces transgredir la ley. A causa de su expresión en el espacio público (sin las garantías de un hogar ni de los lazos sociales de sus barrios de origen) las madres entrevistadas temían que la vida en el campamento fueran también factores de amenaza social para sus hijos.

\title{
Conclusiones
}

Nuestro análisis in situ de los espacios públicos después de un desastre nos ha permitido reflexionar respecto del papel central de las mujeres en su construcción. Los espacios físicos concebidos o formados por ellas nos llevan a mirar a las mujeres no sólo como víctimas de desigualdades o situaciones desventajosas, sino también como sujetos dotados de capacidades y habilidades singulares, otorgando a esta categoría de la población un rol como pilar de la resiliencia y la reconstrucción después del desastre natural en Chile. 
Karen Andersen - Laura Rodríguez - Cristóbal Balbontín: Las mujeres en la reconstrucción del espacio público post-catástrofe socio-natural en Dichato, Chile (2010-2013). Una aproximación hermenéutica desde el mito de Antígona, Izquierdas, 49, abril 2020:725-742

Queremos destacar el espacio público femenino chileno como un espacio distinto del espacio tradicionalmente masculino. Siendo este espacio femenino, un espacio nacido de una demanda urgente, no teme alterar los límites entre lo público y lo privado, ni inyectar temas íntimos en el corazón de la arena pública cuando esto es necesario, refrendando con esto el mito de Antígona. La adaptación de las mujeres a las ciudades basada en principios masculinos y en sociedades donde sufren discriminación, hace que los espacios públicos femeninos cotidianos destaquen como espacios de protección y cuidado vis-à-vis de la comunidad y familia.

Se observa como la configuración física de los espacios públicos contribuye a la resiliencia necesaria después de un desastre. De nuestras entrevistas, notamos la importancia de espacios de encuentro y actividades grupales para mujeres, quienes también fueron las principales líderes de estas reuniones para promover el intercambio entre vecinos. La identidad femenina, como ha sido construida a través de una historia de opresión patriarcal, determina expectativas, oportunidades y responsabilidades diferentes entre hombres y mujeres. Además, determina los recursos y las capacidades para enfrentar un desastre y recuperarse.

A partir de nuestra investigación en el campamento "El Molino" de Dichato, pudimos comprobar que esta determinación de identidad de género no es tal. Así, en estas situaciones, la mujer abandona los roles establecidos, abriendo los espacios públicos de participación. La precariedad del ámbito de lo privado en una situación de emergencia post desastre potencia la participación de la mujer en los asuntos públicos. Incluso si las acciones están, inicialmente, limitadas a espacios públicos no reconocidos por el programa de emergencias y reconstrucción, gradualmente comienzan a permear los espacios públicos tradicionales, aportando diagnósticos y soluciones desde procesos participativos autoconvocados. Así, los grupos de habitantes a partir de estas reuniones auto-convocadas lograron dialogar y hacer efectivas algunas de sus demandas en relación a las condiciones del campamento y del proceso de reconstrucción de viviendas definitivas.

El artículo refleja los roles femeninos emergentes en estas condiciones hostiles, destacando la participación comunitaria de las mujeres para enfrentar la emergencia. Hay una complementariedad entre los objetivos de mitigar los desastres naturales y promover la participación y el liderazgo de la mujer, por lo que incluirlas desde un inicio en la planificación de los procesos participativos sería beneficioso. A partir de estos resultados, creemos que una participación ciudadana exitosa, debe ser capaz de incluir las formas de organización de los espacios públicos alternativos o cotidianos, ya que son estos los que representan con más exactitud las demandas, debilidades y fortalezas de una comunidad.

Estos espacios públicos abiertos de forma paralela a los espacios públicos oficiales, corresponden a lo que Oskar Negt ${ }^{36}$ llamará espacio público oposicional. Este tipo de espacio nace al interior de grupos excluidos de los de los espacios de visibilidad oficiales. Una de las ventajas de estos espacios es ser críticos y propositivos frente a las decisiones que se toman en el espacio público oficial. Negt va a destacar el aporte de estos espacios en la lucha por la reivindicación de la experiencia dentro de las decisiones tomadas desde la razón pura en el espacio público burgués de Habermas. ${ }^{37}$

Concluimos que la serie de acciones e intervenciones de las mujeres víctimas, motivadas por la situación de precariedad se transforman en sí en una red de producción de un tipo particular de espacio. Este espacio nace de forma espontánea y paralela a los espacios públicos oficiales y está dotado de características particulares. El origen de estas acciones espontanea es producto de la relación sensible: afectiva, memorial, social y sensorial con los espacios de vida. Cuando las relaciones sensibles entre el habitante y su lugar no son tomadas en cuenta por los planes de reconstrucción urbana, ellos revindican su existencia manifestándose de formas alternativas. Estos aspectos sensibles van a aparecer en las tres

36Oskar Negt, L'espace public oppositionnel, Paris, Editions Payot. 2007; Karen Andersen, “Una reflexión desde los espacios públicos a la creación colectiva del espacio", Revista de Arquitectura Vol. 21 · N 31, 2016.

${ }^{37}$ Jürgen Habermas, L'espace public, Paris, Editions Payot, 1992. 
Karen Andersen - Laura Rodríguez - Cristóbal Balbontín: Las mujeres en la reconstrucción del espacio público post-catástrofe socio-natural en Dichato, Chile (2010-2013). Una aproximación hermenéutica desde el mito de Antígona, Izquierdas, 49, abril 2020:725-742

dimensiones del espacio conceptualizado por Lefebvre: en las prácticas de los espacios (lo percibido), en las representaciones del espacio (lo concebido) y en los espacios de representación (lo vivido). ${ }^{38}$

Estas manifestaciones alternativas y espontáneas caracterizan estos espacios vividos y construidos por los habitantes. Estos espacios femeninos creados en el contexto de un campamento de emergencia conforman formas de resistencia vis a vis de los procesos de modificación de los espacios y de los ambientes, así como frente a condiciones de vida poco favorables. Son también espacios de producción y creación colectiva. Conforman, además, formas de resiliencia necesarias para salir de la situación de emergencia y comenzar la reconstrucción de sus vidas. Estas manifestaciones nacen de una necesidad espontánea de los habitantes en reacción a una experiencia de vida, pueden ser más o menos voluntarias, así como más o menos organizadas.

Por último, también es importante enfatizar la distancia desde el terremoto del 2010 en Chile y el año en que vivimos. Eso da cuenta también de la fragilidad del énfasis en los estudios de género; la falta de seguridad en que, como dice Linda McDowell serán tomados en serio las distintas perspectivas. Las mujeres cumplen un rol singular y también plural en nuestra sociedad y hasta ahora han sido incorpóreas, sin opinión. Este artículo propone incorporar esa visión ausente, señalando algunos aspectos con los cuales algunos lectores podrían sentirse identificados y finalmente representados en estos escritos.

\section{Bibliografía}

Allan, Penny y Bryant, Martin, The Critical Role of Open Space in Earthquake Recovery: A Case Study, Paper $n^{\circ} 34$ NZSEE Conference (New Zealand Society for Earthquake Engineering Inc), Victoria University of Wellington, Wellington, New Zealand. URL: http://www.nzsee.org.nz/db/2010/ . 2010.

Amoros, Celia, Feminismo, igualdad y diferencia, México, UNAM, PUEG. 1994.

Amphoux, Pascal, Thibaud, Jean-Paul y Chelkoff, Gregoire, (dir.), Ambiances en débats, Grenoble, Editions la Croise. 2004.

Andersen, Karen, "Una reflexión desde los espacios públicos a la creación colectiva del espacio", Revista de Arquitectura Vol. 21 · N 31. 2016.

Andía Pérez, Bethsabé, "Lo Personal Es Político: Una Lectura De Lo Público Y Lo Privado", Boletín Generando del Instituto Runa de Desarrollo y Estudios sobre Género, Año 1, n¹0, Lima. 2007.

Arendt, Hannah, Condition de l'homme moderne, Paris, Editions Calmann-Levy. 1983.

Berroeta, Hector, Ramoneda, Álvaro, Opazo, Luis, Sentido de comunidad, participación y apego de lugar en comunidades desplazadas y no desplazadas post desastres: Chaitén y Constitución. Universitas Psychologica, 14(4), 2015. Pp.1221-1233. https://doi.org/10.11144/Javeriana.up14-4.scpa .

Bondi, Liz, "Making connections and thinking through emotions: between geography and psychotherapy". Trans Inst Br Geogr NS 30. 2005. Pp. 433-448

Buró de Prevención de Crisis y Recuperación, Programa de las Naciones Unidas para el Desarrollo (PNUD), Genero y Desastres. URL: http://www.undp.org. 2010.

38 Henri Lefebrve, La production de l'espace, Paris, Editions Anthropos, 2000. 
Karen Andersen - Laura Rodríguez - Cristóbal Balbontín: Las mujeres en la reconstrucción del espacio público post-catástrofe socio-natural en Dichato, Chile (2010-2013). Una aproximación hermenéutica desde el mito de Antígona, Izquierdas, 49, abril 2020:725-742

Butler, Judith, "Bodily Vulnerability, Coalitions, and Street Politics". Critical Studies Vol. 37, 2014, p99119.

Butler, Judith. El Grito de Antígona. Barcelona; El Roure Editorial, 2001.

Caldarone, Rosaria, "Subjectivity and Femininity: Reading Antigone". CR: The New Centennial Review, 17(2), 2017. Pp. 1-14. https://doi.org/10.14321/crnewcentrevi.17.2.0001

Calhoun, Craig, (dir.), Habermas and the Public Sphere, Cambridge, The MIT Press. 1992.

Carstens, Carolina y Huepe, Gabriela, Rememorando Dichato. Un paseo porpaisajes y relatos. [Concepción], Santiago de Chile, Consejo Nacional de la Cultura y las Artes. 2011.

Cefai, Daniel, L'Enquête de terrain, Paris, Editions La Decouverte. 2003.

Chombart de Lauwe, Paul-Henry, La vie quotidienne des familles ouvrières, Paris, Editions C.N.R.S. 1956.

Coanus, Thierry y Perouse, Jean-François, (dir.), Villes et risques Regards croisés sur quelques cités " en danger», Paris, Editions Economica. 2006.

Cornejo, Consuelo, Oficina Nacional de Emergencia Ministerio del Interior y Seguridad Pública División de Protección Civil, Reducción del Riesgo de Desastre con Enfoque de Género. Género, Desastres y Gestión. Programa de Mejoramiento de la Gestión Sistema de Equidad de Género. URL: www.onemi.cl. 2012.

De Certeau, Michel, Linvention du quotidien, Tome 1 : Arts de faire, Paris, Editions Gallimard. 1990.

De Certeau, Michel, Giard, Lucie, y Mayol, Pierre, L'invention du quotidien, Tome 2 : Habiter, cuisiner, Paris, Editions Gallimard. 1994.

De Beauvoir, Simone, The Second Sex. London: Jonathan Cape, 1956.

Detraz, Nicole, Peksen, Durson, "In the Aftermath of Earth, Wind, and Fire: Natural Disasters and Respect for Women's Rights”. Human Rights Review, 18(2), $2017 . \quad$ Pp.151-170. https://doi.org/10.1007/s12142-016-0440-4

FLACSO, Más Voces, Más democracia: Propuestas para la participación ciudadana. Santiago, Chile. 2005.

Fraser, Nancy, "Rethinking the Public Sphere: A Contribution to the Critique of Actually Existing Democracy", Social Text, No. 25/26, pp. 56-80 Published by: Duke University Press Stable. URL: http://www.jstor.org/stable/466240 . 1990.

García Acosta, Virginia, (dir.), "Historia y Desastres en América Latina: volumen II, Lima", La RED de Estudios Sociales en Prevención de Desastres en América Latina, CIESAS, ITDG. 1997.

Giannini, Humberto, La 'réflexion' quotidienne. Vers une archéologie de l'expérience, Aix en Provence, Editions Alinea. 1992.

Goffman, Erving, Behavior in Public Places, New York, The Free Press. 1963. 
Karen Andersen - Laura Rodríguez - Cristóbal Balbontín: Las mujeres en la reconstrucción del espacio público post-catástrofe socio-natural en Dichato, Chile (2010-2013). Una aproximación hermenéutica desde el mito de Antígona, Izquierdas, 49, abril 2020:725-742

Goffman, Erving, Les rites d'interaction, Paris, Editions de Minuit. 1974.

González-Muzzio, Claudia, "El rol del lugar y el capital social en la resiliencia comunitaria postdesastre. Aproximaciones mediante un estudio de caso después del terremoto del 27/F", Revista EURE. Vol 39 , n'117, pp. 25-48. 2013.

Grosjean, Michèle y Thibaud, Jean-Paul, (ed), L'espace public en méthodes, Editions Parenthèses. 2008.

Habermas, Jürgen, L'espace public, Paris, Editions Payot. 1992.

Hegel, G.W.F. Werke 3: Phänomenologie des Geistes. Frankfurt, Suhrkamp.1989

Heidegger, Martin, Sein und Zeit, Tubingen: Niemeyer. 1993,(1926).

Honig, Bonnie. Antigone Interrupted. New York: Cambridge University Press, 2013.

Irigaray, Luce. Speculum of the Other Woman. New York: Cornell University Press, 1987.

Lefebrve, Henri, La production de l'espace, Paris, Editions Anthropos. 2000.

Ministerio de Planificación, PNUD Chile, Encuesta Post Terremoto: Principales resultados. Efectos en la calidad de vida de la población afectada por el terremoto/tsunami, URL: http://www.ministeriodesarrollosocial.gob.cl/encuesta-postterremoto/documentos/informe-encuestapost-terremoto.pdf . 2010.

Negri, Antonio y Hardt, Michael, Multitude. Guerre et démocratie à l'âge de l'Empire, Paris, Editions La Découverte. 2004.

Negt Oskar, L'espace public oppositionnel, Paris, Editions Payot. 2007.

Noschis Kaj, Signification affective du quartier, Lausanne, Editions Librairies Méridiens. 2011.

Observatorio del Ministerio De Desarrollo Social, Gobierno de Chile, Trabajo y Mujer. (URL: http://observatorio.ministeriodesarrollosocial.gob.cl. 2011

ONU, Bureau pour la Prévention des Crises et le Redressement, Programme des Nations Unies pour le Développement, Genre et Catastrophe. URL: www.undp.org. 2010.

Ricoeur, Paul, De l'interpretation, Paris, Editions Seuil. 1965.

Romero Toledo, Hugo, Romero Aravena, Hugo. "Ecología política de los desastres: vulnerabilidad, exclusión socio-territorial y erupciones volcánicas en la patagonia chilena". Magallania (Punta Arenas), 43(3), 2015. Pp.7-26. https://dx.doi.org/10.4067/S0718-22442015000300002

Roulleau-Berger, Laurence, La rue, miroir des peurs et de solidarités, Paris, Éditions PUF. 2004. 
Karen Andersen - Laura Rodríguez - Cristóbal Balbontín: Las mujeres en la reconstrucción del espacio público post-catástrofe socio-natural en Dichato, Chile (2010-2013). Una aproximación hermenéutica desde el mito de Antígona, Izquierdas, 49, abril 2020:725-742

Rousseau, Jean-Jaques, Émile ou De l'éducation, Paris, Garnier, 1961.

Salazar, Gabriel, "De la participación ciudadana: capital social constante y capital social variable (Explorando senderos trans-liberales)", Proposiciones Vol. 28. Ediciones SUR, Santiago de Chile. URL: http://www.sitiosur.cl/r.php?id=268. 1999.

Sandoval, Alejandra y Aravena, Susana, (Coord), Dichato, nuestra tierra. Ediciones SUR, Santiago de Chile. URL: http://www.sitiosur.cl/r.php?id=922\#sthash.Z1TILY6F.dpuf. 2011

SERNAM, Balance Gestion Integral 2010, 2010.

Universidad de Concepción, Informe Ambiental, Calidad de Agua para Consumo Humano y Manejo de Residuos Sólidos Domésticos en Aldeas de Emergencia de Coronel, Dichato y Lota. URL: www.eula.cl/doc/Informe\%20Aldeas.pdf. 2010.

WHO, Department of Gender, Women and Health, Gender and Health in Natural Disaster. URL : http://www.who.int/gender-equity-rights/en/. 2005. 